\title{
Comparison of approval procedures for medical devices in Europe and the USA
}

https://doi.org/10.1515/cdbme-2019-0152

\begin{abstract}
Important achievements such as the discovery of penicillin increased life expectancy in the 19th century. At the beginning of the 20th century, the influence of technical equipment and biomedical research led to a further leap in life expectancy. The use of computer devices with sophisticated software also improved the quality of life. This is linked to the fact that all these achievements have now led to the emergence of one of the largest import and export markets in the USA, alongside the european union (EU), which is an association of European states. In order to guarantee a uniform quality of this abundance of different products and therapies, there are worldwide corresponding regulations, to which all manufacturers must adhere. The entry into force of these regulations, with the associated approval and testing obligations for each individual medical device, ensures that a consistently high quality of products is used on patients. Of course, high quality as well as high functionality and safety levels of medical products are a must. However, the implementation of these regulations is realized differently in the various markets, as there is no global harmonization. This work focuses on approval procedures for medical devices in Europe (EU) and the USA and compares significant differences in the approval procedures, which are shown as a graphical comparison. An example is an approval of medical devices by the U.S. Food and Drug Administration (FDA), which is an US government agency. In Europe (EU) there is a manufacturer's declaration of conformity for the approval.
\end{abstract}

Keywords: Approval medical devices, FDA, MDR

\section{Introduction}

Today, medical devices are an essential part of our daily lives. Whether it is a small plaster or an implant, we speak of medical products. The reason why medical devices can be classified into so many groups and why there are so many of them today depends on the constant development of the products. It

\footnotetext{
*Corresponding author: Marcel Vila Wagner, Technische Hochschule Mittelhessen (THM), FB Life Science Engineering (LSE), Institut für Biomedizinische Technik (IBMT), Wiesenstr. 14, 35390 Gießen, Germany, e-mail: marcel.vila.wagner@Ise.thm.de Thomas Schanze, IBMT, Gießen, Germany, e-mail: thomas.schanze@lse.thm.de
}

is the goal of every medical device manufacturer to maintain a constant quality and to guarantee a certain safety and effectiveness. In order to be able to guarantee these high demands on quality, manufacturers are obliged to comply with certain conditions and regulations, which are subject to constant monitoring. The intention is to assure the safety of the products for patients, users and third parties. In order for a medical device to be marketed, each country has implemented its own approval requirements and approval procedures for medical devices. In the EU, manufacturers must prove the conformity of their medical devices themselves and, if necessary, cooperate with a Notified Body (NB) which is not an authority. In the USA, the FDA is an authority that regulates the approval of medical devices. Nevertheless, in the USA and the EU, the basic idea behind the approval of medical devices is based on the classification of medical devices into risk classes. Each risk class has a different risk and has corresponding damage effects. Because of the high requirements on safety, efficacy and quality for medical devices, manufacturers of the USA have established themselves as a cooperation partner in the field of import and export of medical devices for the EU. As a result, there is a strong trade in medical devices between the USA and the EU [1]. This work is intended to provide an insight into the approval procedures of the USA and EU. The key points relate to the distinction between the classification of medical devices in the EU and the USA, to which guidelines and standards the USA refers, and which are used in the EU. Another aspect is how the government is involved in the approval of medical devices. Furthermore, the differences between the approval procedures and approval processes are pointed out, presented and finally discussed.

\section{Classification}

In the USA and the EU, the classification of medical devices into risk classes is the basis for marketing approval. Each medical device is assigned to a certain risk class depending on its invasiveness. According to the risk class, a different procedure is used or other regulations with corresponding requirements are complied in order to obtain marketing approval. The classification of medical devices is used for constant safety and verification of the efficacy of medical devices. The higher the risk class, the stricter the requirements and controls of a prod- 
uct. The USA has implemented a database through the FDA which classifies approximately 1700 different types of medical devices in 18 different panels [2]. According to the FDA, medical devices are classified in risk class I (e.g. tongue depressor) with a low risk. Products with a medium risk in class II (e.g. infusion pumps) and products with a high risk in class III (e.g. replacement of heart valves). In the EU, medical devices are also classified into three risk classes. The difference here is that risk class I is divided into a class for sterile media products (Is) and into medical devices with measuring function (Im) [3]. With the implementation of Medical Device Regulation (MDR), the risk class for reusable surgical instruments ( $\left.{ }^{*}\right)$ has also been added. In addition, in 2020 the EUDAMED database will be implemented, which has been in development since 2010. This database is similar to the one in the USA [3]. An important goal of the so called European Database on Medical Devices (EUDAMED) is to improve constantly market surveillance [3], [8]. In addition, risk class II in the EU is divided into IIa and IIb. How the risk classes are classified is clearly prescribed in Annex VIII of the MDR. A distinction is made between risk class I with the lowest risk (e.g. plaster), risk class IIa with a medium risk (e.g. hearing devices) and class IIb with a high risk (e.g. X-ray devices). The products in risk class III (e.g. knee and hip implants) have the highest risk [2],[3].

\section{Procedure for medical device approval}

In the USA, the FDA has established approval processes and procedures for the market approval of medical devices (see Figure 1). Depending on whether a new medical device can be classified according to the Code of Federal Regulations (21CRF) or not, other procedures are used. If a new device can be classified under $21 \mathrm{CRF}$, it can pass the premarket notification $(510(\mathrm{k}))$, or the premarket approval (PMA). The $510(\mathrm{k})$ procedure is applied if the product falls into risk class I or risk class II. Medical devices in risk class III pass through the PMA procedure. If a new device cannot be classified according to 21CRF, it automatically falls into risk class III. However, if the manufacturer submits a de novo application, the FDA can downgrade the device to risk class I or II. The $510(\mathrm{k})$ procedure, is an equivalence procedure. "The $510(\mathrm{k})$ process has become a major component of medical-device regulation in the United States. Thousands of devices are cleared via the $510(\mathrm{k})$ process each year - about one-third of devices entering the market. The remaining devices are exempt from any premarket review $(67 \%)$ or enter the market by the premarket approval (PMA) pathway (1\%)" [4]. In this procedure, the

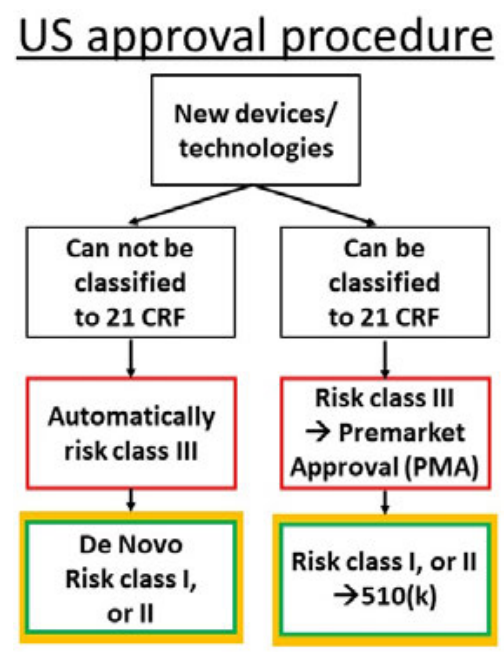

Fig. 1: Approval procedure in the USA. New non-classifiable devices are automatically risk class III unless the manufacturer chooses the de novo procedure where the device is classified by the FDA as class I or II. New classifiable risk class III devices must pass through the PMA and risk class I or II devices must go through the $510(\mathrm{k})$ procedure [2].

intended purpose and the specific technical characteristics of the device and the safety and efficacy are compared with existing devices (predicat device) that are already on the market. Most devices in risk classes I and II undergo this procedure. Devices classified in risk class III must undergo the premarket approval (PMA) defined in 21CRF Part 814. The PMA process is a regulatory and scientific procedure to verify the safety and efficacy of medical devices. Because the risk class III products have a very high risk level, the FDA has implemented the strictest procedure for the approval of medical devices with the PMA. Premarket approval is based on the fact that the safety and efficacy of the product is guaranteed at all times with sufficient clinical studies and scientific evidence. If medical devices cannot be compared with any predicate device because it is not yet on the market, the device will automatically be classified in risk class III. With the de novo procedure, a procedure was implemented by the FDA where the FDA assigns a risk class to new devices or products for which no $510(\mathrm{k})$ or PMA procedure can be applied [2], [9]. Without a CE mark it is impossible in the EU to apply a medical device on the market. In order to obtain a CE mark, certain requirements and cooperation with the NB are required. Every manufacturer must submit a declaration of conformity for his medical device. The conformity assessment is different depending on risk class I, IIa, IIb and III and is described in chapter five of the MDR. With the corresponding conformity assessment, manufacturers establish the essential requirements of the European Directive by going through a conformity assessment procedure [5]. The path to CE marking can be divided into four phases (see Fig- 


\section{EU approval of medical devices}

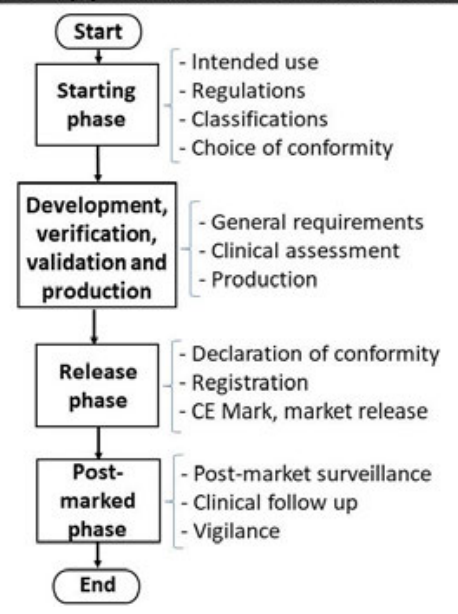

Fig. 2: In order to be able to sell medical products in Europe (EU), a CE mark is required. In order to obtain this, an approval procedure must be carried out. This can be divided into the start phase, development and production phase, release phase and post market phase [5], [8].

ure 2). At the beginning, the manufacturer must define the intended use of his product in order to carry out a corresponding risk classification according to the European (EU) regulations. Depending on the risk class, a different conformity assessment procedure is carried out. Next, the essential requirements specified by the standards must be complied during the production process. In order to be able to sell the product on the market, cooperation with a NB is necessary. Products that can be assigned to risk class I can be treated differently. Manufacturer and distributors can declare the conformity of their product without a NB. From risk class IIb onwards, NB still work together with the Medical Device Coordination Group (MDCG). After the declaration of conformity of the product, the NB issues the CE mark and the manufacturer may sell his product on the European market. After market approval, the manufacturer is required to carry out an active post market surveillance on a regular basis. In addition to post-market surveillance, a clinical follow up and vigilance must be implemented [3], [5].

\section{Gouvernment involvment}

In the USA the corresponding approval procedures, or rejection of all risk classes of medical devices (I, II, III), the approval or dissaproval is carried out directly by the FDA. If an approval is granted, this is applicable for all states of the USA. If a medical device consists of a combination of several components (e.g. drug/device), the FDA's decision assigns the medical device to the corresponding area of responsibility of the FDA. For all three risk classes, general controls are mandatory as regulatory requirements. General controls include inspections such as the registration of medical device manufacturers or the listing of medical devices by/with the FDA. Risk classes II and III require more control by the FDA. In addition to general controls, there are also special controls and premarket approvals. Special controls include the verification of performance standards and special markings and labelling. For risk class III products, premarket approval (PMA) controls also apply. This application contains the strictest requirements of the FDA. The PMA requires manufacturers to provide evidence of the safety and efficacy of their product through clinical trials. The FDA provides a variety of guidance documents. These documents can all be found in a database and they can be differentiated according to topics. These guidance documents are addressed to the industry and also to the FDA and reflect in detail what kind of requirements one has to consider. These documents contain, for example, the production of audit reports or technical documents [2], [9]. In the EU, the approval of medical devices is not determined by an authority, as in the USA, but by declaration of conformity of the manufacturers must be, if necessary, done with the cooperation of a NB. From risk class II onwards, the inclusion of a NB is required in order to obtain market approval. With the implementation of the MDR, a scrutiny procedure was introduced. The approval procedure for products of eisk class IIb or higher is carried out via a cooperation of the manufacturers with a $\mathrm{NB}$ and an expert commission MDCG. In the EU, harmonized standards are available for medical devices, which are published by the European Committee for Standardization (CEN). These harmonized standards include, for example, the application of risk management to medical devices (ISO EN 13485). These standards can be used as a tool to achieve compliance with certain requirements. Since, in contrast to the American guidance documents, these standards are sometimes kept fairly open, the result for the manufacturers is an individual implementation of the requirements. For example, ISO EN 13485 Chapter 4.1.2 requires a risk-based approach. Although a draft is presented, explicit requirements for manufacturers are not given [6].

\section{Results}

Some differences become apparent with the approval of medical devices in the USA and the EU (see Figure 3). However, there is "no evidence of superiority of a system" [7] in the comparison of the USA and the EU. Basically, there are few similarities, but these have little in common only in the main features and basic ideas, such as in the classification and application of guidelines, which in some cases must be interpreted 
more openly in the EU. In the USA, approval is controlled by one authority, the FDA. In the EU, manufacturers must accomplish a declaration of conformity. If necessary with the involvement and cooperation of a NB, which is not an authority. With the premarket notification, or $510(\mathrm{k})$ procedure, the FDA has implemented a procedure that does not exist in Europe.

\section{Differences USA/EU}

\begin{tabular}{|l|l|}
\hline \multicolumn{1}{|c|}{ USA } & \multicolumn{1}{|c|}{ EU } \\
\hline $\begin{array}{l}\text { Approval of medical devices by a federal } \\
\text { agency (FDA) }\end{array}$ & $\begin{array}{l}\text { Approval with the declaration of } \\
\text { conformity via the manufacturer with } \\
\text { notified body }\end{array}$ \\
\hline $\begin{array}{l}\mathbf{5 1 0 ( k )} \text { Method based on proof of } \\
\text { equivalence of medical devices }\end{array}$ & $\begin{array}{l}\text { No procedure based on the proof of } \\
\text { equivalence is implemented in Europe }\end{array}$ \\
\hline $\begin{array}{l}\text { Classification of the medical devices in } \\
\text { classes is made by the agency in different } \\
\text { panels }\end{array}$ & $\begin{array}{l}\text { Classification of the medical devices with } \\
\text { relevant approval requirements is } \\
\text { determined by rules and regulations }\end{array}$ \\
\hline $\begin{array}{l}\text { Clearly defined guidance documents of } \\
\text { the FDA are relevant for the proof of the } \\
\text { requirements }\end{array}$ & $\begin{array}{l}\text { The proof is based on harmonized } \\
\text { standards and MEDDEV documents, which } \\
\text { give the manufacturers custom solutions }\end{array}$ \\
\hline
\end{tabular}

Fig. 3: Differences of the approval of medical devices in the USA and Europa (EU).

The $510(\mathrm{k})$ procedure is based on the fact that a comparable product has already been approved and thus the essential relevant questions regarding safety and performance are answered, so that the approval concentrates on the proof of equivalence. Another difference is that in the EU the classification of medical devices and the associated approval requirements are determined by a set of rules. In the USA, the classification of products depends on the FDAs division into a certain panel (database). The proof of conformity in the EU is based on the inclusion of harmonized standards and medical devices documents (MEDDEV), which in certain parts impose an individual design of the requirement on the manufacturers. In the USA clearly defined guidance documents of the authority are decisive.

\section{Discussion}

The analysis of the different approval processes clearly shows that it is impossible to make a statement about whether one system (USA or EU) is superior to the other. For both parties, the basic idea is based on the classification of medical devices into risk classes. This is because the harmonized standards give the manufacturer more room for interpretation than the guidance documents in the USA do, but in the EU, this means that each manufacturer has to find its own individual solution and, finally, to fulfill the requirements. As a consequence, small and medium-sized enterprises (KMU) have to master a process, which requires high financial and human resources to adapt to the corresponding freedoms of the EU. However, the impression arises that documents and procedures at the FDA are more clearly described and are easier for small companies to handle. Thus one could take this system of the solution development of the FDA as a consideration or assistance. A better harmonisation between the USA and the EU would have the advantage of simplifying the development and approval of medical devices for many medical product manufacturers, especially for KMU. Through the uniform standardization of documents and solutions, it could be possible for NBs to identify deviations more easily and quickly, since there are no longer any real individual company-specific documents, but an equivalent equality of documents in all companies.

Author Statement Research funding: The author state no funding involved. Conflict of interest: Authors state no conflict of interest.

\section{References}

[1] Bundesministerium für Wirtschaft und Gesundheit. Fakten und Zahlen, Ausgabe 2016. „Sonderthema, Medizinprodukte und Medizintechnik".

[2] The Food and Drug Administration (FDA). „Classify Your Medical Device“. Abgerufen von https://www.fda.gov/medicaldevices/overview-device-regulation/classify-your-medicaldevice

[3] Medical Device Regulation 2017/745

[4] Dr. David R. Challoner Vice President for Health Affairs, Emeritus University of Florida And Chair, Committee on the Public Health Effectiveness of the FDA 510(k) Clearance Process Institute of Medicine of The National Academies." Medical Devices and the Public's Health: The FDA 510(k) Clearance Process at 35 Years"

[5] Johner Institut. „Der Weg zum CE-Zeichen“. Abgerufen von https://www.johner-institut.de/blog/iec-62304-medizinischesoftware/ce-zeichen-so-bestehen-sie-das-ce-audit/

[6] Medizinprodukte - Qualitätsmanagementsysteme - Anforderungen für regulatorische Zwecke (ISO 13485:2016); Deutsche Fassung EN ISO 13485:2016

[7] BVMed Gesundheit gestalten. (2015, 26 Juni). [Pressemitteilung 53/15, Köln]. MedizinprodukteZulassungsexperte zu Europa vs. USA: "Keine Anhaltspunkte für die Überlegenheit eines Systems" Abgerufen von https://www.bvmed.de/de/bvmed/presse/pressemeldungen/medizinprodukte-zulassungsexperte-zu-europa-vs.-usakeine-anhaltspunkte-fuer-die-ueberlegenheit-eines-systems

[8] BVMed Gesundheit gestalten. (2016, 20 Januar). [Hintergrundartikel BVMed] "Der lange Weg eines Medizinprodukts von der Idee bis zur Anwenung am Patienten"

[9] Institute of Medicine 2011. "Medical Devices and the Public's Health: The FDA 510 (k) Cleatance Process at 35 Years." Washington, DC: The National Academies Press. 